Oriolus Revista Científica, ISSN: 2215-6100.1(1). Julio, 2020, pp 27-38

Alpízar Vaglio

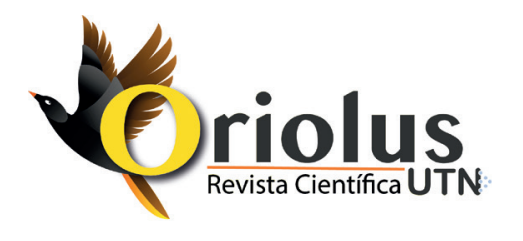

Oriolus Revista Científica

ISSN: 2215-6100

revista.cientifica.oriolus@utn.ac.cr

Universidad Técnica Nacional

\title{
Ecuación práctica para cubicar madera
}

\section{Practical equation for cubing wood}

Edwin Alpízar Vaglio

Ecuación práctica para cubicar madera

Oriolus Revista Científica, Vol.1, N. ${ }^{\circ}$ 1, 2020

Universidad Técnica Nacional

Disponible en: http://revistas.utn.ac.cr/index.php/oriolus

Bibliografía:

Alpízar Vaglio, Edwin. "Ecuación práctica para cubicar madera". Revista Científica Oriolus 1, N. 1 (2020): 27-38.

Esta obra esta bajo una Licencia de Creative Commons Atribución NoComercial-SinDerivar Internacional 
Oriolus Revista Científica, ISSN: 2215-6100.1 (1). Julio, 2020, pp 27-38

Alpízar Vaglio

\title{
Ecuación práctica para cubicar madera
}

\author{
Practical equation for cubing wood
}

Edwin Alpízar Vaglio

Universidad Técnica Nacional, Costa Rica

ealpizar@utn.ac.cr

(iD) https://orcid.org/0000-0002-4171-2827
Recepción: 29 de abril del 2019

Aprobación: 11 de febrero del 2020

\section{Resumen}

Se presenta la situación en torno a la Ley sobre el uso obligatorio del Sistema Internacional de Unidades, que deja como ilegal la cubicación de madera con el método de la "Pulgada Maderera Tica" (PMT). A pesar de los esfuerzos de la academia por proporcionar ecuaciones de cubicación, este sistema se mantiene vigente en la actualidad. Se explican de manera general las ecuaciones de más uso en Costa Rica. Asimismo, se sugiere que estas ecuaciones generadas por la academia no son utilizadas debido a la dificultad técnica de su aplicación por parte de los usuarios madereros y por la sobreestimación que hacen al considerar la totalidad del volumen y no el volumen comercial. Se propone una ecuación práctica que se basa en cubicar la madera en troza, sin considerar su redondez, sino que se estima el volumen comercial que se obtendría con el aserrío primario. Se demuestra que las ecuaciones al mecate y del cilindro sobreestima el volumen en más del $19 \%$. Finalmente, se sugiere que la ecuación propuesta puede ser aplicada para árboles en pie, con lo cual se debe considerar la forma del fuste y su longitud, de modo que se defina un factor de ajuste al dap.

Palabras claves: modelos alométricos, cubicación, trozas, volumen, Smalian.

\begin{abstract}
The situation is presented around the law on the compulsory use of the International System of Units, which makes illegal the cubing of wood with the method of the "Pulgada Maderera Tica" (PMT). In spide of the efforts of the academy to provide cubing equations, this system remains in effect today. The equations of most use in Costa Rica are explained in a general way. The author suggests that these equations generated by the academy are not used due to the technical difficulty of their application by logging users and the over estimation they make when considering the whole volume and not the commercial volume. A practical equation is proposed that is based on cubing the wood in logs, without considering its roundness, but estimating the commercial volume that would be obtained with primary sawing. It is shown that the equations to the rope and the cylinder on estimated the volume in more than $19 \%$. Finally, it is suggested that the proposed equation can be applied to standing trees, for which the form of the stem and its length should be considered, so that an adjustment factor to the dap is defined.
\end{abstract}

Keywords: allometric models, cubing, logs, volume, Smalian. 
Oriolus Revista Científica, ISSN: 2215-6100.1 (1). Julio, 2020, pp 27-38

Alpízar Vaglio

\section{Introducción}

La madera es el principal producto comercial que se obtiene de los árboles en los bosques, las plantaciones y de forma aislada en los potreros y otros arreglos arbóreos. Por lo que es un recurso renovable que tiene infinidad de usos. La economía de un país podría verse beneficiada con la producción de madera, la manufactura de productos y su comercialización. Por ello, es importante su cuantificación; práctica que usualmente se le denomina cubicación de madera.

La cubicación de la madera presenta un reto, por cuanto es necesario estimarla cuando los árboles están en pie, de modo que se pueda determinar el potencial de un bosque o plantación forestal y así, permita tomar decisiones para su manejo productivo y sostenible.

Por otro lado, para la cubicación de madera en troza, el sector maderero en Costa Rica ha utilizado el método de mecate, cuyas unidades son conocidas como la Pulgada Madera Tica (PMT); es común que se trate de relacionar la ecuación de la PMT para madera en troza, con la cubicación de la madera en pie.

La promulgación de la Ley N. 5292 en el año 1973 estableció como obligatorio el uso del Sistema Internacional de Unidades (ITCR/DGF, s.f.); basado en el Sistema Métrico Decimal, en sus unidades básicas, derivadas y suplementadas de medición (Costa Rica, 1973). Esta ley plantea una situación en el sector forestal, por cuanto el sistema utilizado es el de medición al mecate, el cual utiliza unidades en pulgadas y varas; a partir de esta ley, la medición al mecate es ilegal.

El Estado trató de mantener el sistema al mecate, para lo cual modificó las unidades a través de la promulgación del Decreto Ejecutivo N. 12067-MEIC, en el año 1980, con la Norma Oficial para la Comercialización de madera en Troza y Aserrada en Condición Verde, donde básicamente mantiene la ecuación de cubicación al mecate, pero se cambian las unidades a decímetros de metro (Costa Rica, 1980). Sin embargo, esta iniciativa no prosperó.

Con la implementación de la Ley N. ${ }^{\circ}$ 5292, se comenzaron a utilizar ecuaciones que cubican la madera en metros cúbicos. La primera propuesta fue la ecuación conocida como del MAG, basada en la ecuación del cilindro y ajustado por un factor de forma que corrige la conicidad del fuste. Posteriormente, se utilizaron ecuaciones alómetricas, desarrolladas por investigadores de las universidades nacionales e internacionales.

Las ecuaciones de cubicación de madera en rollo, basadas en la ecuación del cilindro, plantea una condición matemática compleja por parte de los madereros empíricos. Por lo cual, estas ecuaciones para cubicar madera en pie y en rollo, en metros cúbicos, considerando la redondez de los fustes, no han sido aceptadas por el sector que aprovecha y procesa la madera; a pesar de que es obligatorio por ley. Luego de 46 años de promulgada de la ley, el sector maderero continúa utilizando la PMT como unidad de medida. Este divorcio metodológico hace que en el mercado interno de la madera se siga utilizando la PMT; mientras en el mercado internacional y en la academia se utilizan ecuaciones de cubicación de la madera en metros cúbicos, basadas en la forma cilíndrica de los fustes.

Esta obra esta bajo una Licencia de Creative Commons Atribución NoComercial-SinDerivar Internacional 
Oriolus Revista Científica, ISSN: 2215-6100.1 (1). Julio, 2020, pp 27-38

Alpízar Vaglio

Tal como lo plantea Chinchilla y Gómez (2005):

(...) la PMT es un sistema de relativa sencillez y por todos conocido, mientras que el sistema métrico decimal es inaplicable, puesto que las máquinas de aserrío existentes en el país se encuentran graduadas en pulgadas y para poder sacar medidas en centímetros es necesario hacer una transformación a pulgadas. (67)

La anterior es una de las razones para que no se lleve a cabo la cubicación en metros cúbicos. Sin embargo, hay otra razón muy importante, que es la diferencia de volumen entre uno y otro método. Ello se debe a que la ecuación del mecate establece como circunferencia el extremo menor de la troza y para el árbol en pie, la norma forestal consiste en estimar el diámetro a la altura del pecho (a 1,30 m del suelo); es decir, cubica por el extremo mayor de la troza. Esta situación crea desconfianza en los madereros porque sobreestima el volumen de madera.

Actualmente, las ecuaciones de cubicación de madera en pie son complejas y no necesariamente cubican la madera comercial, sino el volumen total que tiene aplicación para determinar biomasa. Por lo que es necesario disponer de una ecuación con mejor aproximación de la madera que será aprovechada, que tenga una aplicación sencilla, pero manteniendo los principios básicos de cubicación de madera, y que sea aplicable a árboles en pie.

De acuerdo con el Proyecto sobre Gobernanza Forestal en la República de Colombia (CARDER, 2013), la cubicación de madera se refiere a la determinación del volumen de árboles y productos forestales, procesados o no, a través de la medición de algunas de sus dimensiones. Cuando los productos tienen formas geométricas definidas se puede saber su volumen exacto, en caso contrario tan solo se podrán hacer estimaciones.

El volumen de madera puede ser total en un individuo arbóreo dentro de un ambiente o ecosistemas, a partir del tocón hasta el ápice de árbol. Es comercial si se excluyen las partes que no son aprovechadas o que poseen secciones con dimensiones inferiores a las establecidas como comerciales (CARDER, 2013).

\section{Ecuaciones usuales en Costa Rica}

De acuerdo con Chinchilla y Gómez (2005) las ecuaciones más utilizadas en Costa Rica para cubicar madera en troza en patio son la de Smalian y la del mecate. También, pueden utilizarse Newton y Huber; sin embargo, son ecuaciones poco prácticas, pues requieren del diámetro medio de la troza. Para árboles en pie, por mucho tiempo, se aplicó la ecuación del MAG. Por su lado, las universidades desarrollaron ecuaciones de cubicación que daban un mejor ajuste a la forma de los fustes de los árboles, considerando su especie y dimensión, utilizando modelos alométricos (Ortiz, 1989). La ecuación más antigua, aún en uso en el país, es la de Loján (1966). A continuación, se describen las ecuaciones más utilizadas en el país:

\section{El método "al mecate"}

Se cree que esta ecuación del mecate es una derivación de la ecuación de Burton, promovida por la Aduana Británica para el cobro de tributos, el cual se basa en una adaptación del sistema Hoppus (Muñoz, 2006), otros autores indican que proviene de una deformación de la ecuación de Doyle (Chinchilla y Gómez, 2005).

Esta obra esta bajo una Licencia de Creative Commons Atribución NoComercial-SinDerivar Internacional 
Oriolus Revista Científica, ISSN: 2215-6100.1(1). Julio, 2020, pp 27-38

Alpízar Vaglio

Flores (1985) en el diagnóstico del sector forestal, describió la ecuación para cubicación de trozas denominada "al mecate". Las unidades son pulgadas madereras ticas (PMT). La ecuación se describe de la siguiente manera:

$$
V=\left(\frac{C}{4}-R\right)^{2} \times \frac{L}{4}
$$

Donde:

C es la circunferencia medida "al mecate", en punta delgada, en pulgadas.

R es un "castigo" que, en promedio, es igual a una pulgada (a veces no se castiga para el pago de transporte) si la troza es sana.

L es el largo en varas, que siendo de 4 varas la norma se convierte en un valor constante igual a 1 (Flores, 1985).

\section{Smalian}

La ecuación de Smalian se aplica para la cubicación de madera en troza y considera la superficie de las dos caras, promediadas y multiplicadas por la longitud de la troza. La ecuación se describe de la siguiente manera:

$$
\mathbf{V}=\left(\frac{\mathbf{S} \mathbf{0}+\mathbf{S} \mathbf{1}}{\mathbf{2}}\right) \times \mathbf{L} \quad(\text { Romahn y Ramírez, 2010) }
$$

Donde:

$\mathbf{V}=$ Volumen en metros cúbicos.

$\mathbf{L}=$ Longitud del fuste $\mathrm{O}$ troza, en metros.

$\mathbf{S}_{0}$ y $\mathbf{S}_{1}=$ Áreas de las secciones transversales extremas del fuste o troza, en metros cuadrados.

\section{Ecuación del MAG}

Se le denominaba la ecuación del MAG porque la Dirección General Forestal pertenecía al Ministerio de Agricultura y Ganadería (MAG). La ecuación se utilizó para cubicar madera en pie, hasta que la Guía para Planes de Manejo en Bosque Natural (DGF, 1990), emitida por la DGF, recomendó no utilizarla y sustituirla por ecuaciones alométricas para grupos de especies, en aquellas en donde no se tuviera una ecuación específica, se utilizaría la ecuación de Loján, la cual será explicada más adelante.

La ecuación del MAG parte de estimar el volumen del cilindro y ajustarlo por un factor de forma. Este factor de forma se determina mediante estudios específicos por especie; para ello el árbol es

Esta obra esta bajo una Licencia de Creative Commons Atribución NoComercial-SinDerivar Internacional 
Oriolus Revista Científica, ISSN: 2215-6100.1(1). Julio, 2020, pp 27-38

Alpízar Vaglio

apeado y se mide el fuste en segmentos, para ajustarse al volumen real; la evaluación se hace por muestreo con valor estadístico, estimando así el volumen total del fuste. El factor de ajuste, llamado también factor de forma o mórfico, es un porcentaje de castigo al volumen del cilindro, definido por la conicidad o ahusamiento del fuste del árbol (Prodan, Peter, Cox y Real, 1997). En el caso de la ecuación del MAG fue definido en 0,7.

\section{Modelos alométricos}

Los modelos alométricos son ecuaciones matemáticas que permiten estimar el volumen de árboles en pie, así como la biomasa y el carbono. Se desarrollan en función de unas pocas variables de fácil medición, tales como el diámetro del tronco a la altura del pecho (dap) y/o la altura total. Para desarrollar un modelo alométrico es necesario definir el ecosistema y la especie de interés. Se hace un muestreo de árboles, los cuales son cortados, medidos y estimado su volumen. Se analizan y se escoge el modelo alométrico de mejor ajuste (Segura y Andrade, 2008).

Una de estas ecuaciones fue desarrollada por Leoncio Loján (1966), la cual ha tenido mucha aplicación en el país. La ecuación es la siguiente:

\section{$\log V=2,03986 \times \log D+\mathbf{0 , 7 7 9} \times \log L$ - 4,07682 (Loján, 1966)}

\section{Donde:}

Log $\mathbf{V}$ es el logaritmo del volumen en metros cúbicos (el volumen se obtiene convirtiendo a antilogaritmo).

D es el diámetro a la altura del pecho en centímetros.

L es la altura en metros.

\section{Aplicación de las ecuaciones de cubicación en Costa Rica}

Luego de más de treinta años de investigaciones por parte del Estado y las universidades, se cuenta con una considerable información sobre ecuaciones para cubicar madera en troza y en árboles en pie. Pero, se sigue utilizando el método al mecate, con unidades de PMT. Esto deja entrever el vacío que se tiene en materia de cubicación de madera, que, a pesar de contar con muchas ecuaciones de cubicación, estas no se aplican. Dos ejemplos que sustentan esta afirmación están en los controles del volumen de madera en los camiones madereros y en la metodología de cubicación utilizada en el Inventario Nacional Forestal.

En el primer ejemplo, la Administración Forestal del Estado exige la cubicación de madera en metros cúbicos; sin embargo, las guías de transporte de madera, que son unos formularios en donde se anotan las trozas que transportan los camiones, además de indicar la especie y sus dimensiones, en el reverso se presenta una tabla de conversiones, de PMT a metros cúbicos, cuyo factor de conversión es de 325 PMT por metro cúbico (SINAC. s.f.).

En el segundo ejemplo, el país realizó el Inventario Nacional Forestal de Costa Rica 2014-2015 (REDD/ CCAD-GIZ, 2015); para hacer las estimaciones de volumen de madera se utilizó la ecuación del cilindro, ajustado por un factor de forma de 0,5.

Esta obra esta bajo una Licencia de Creative Commons Atribución NoComercial-SinDerivar Internacional 
Oriolus Revista Científica, ISSN: 2215-6100.1(1). Julio, 2020, pp 27-38

Alpízar Vaglio

\section{Ecuación propuesta para cubicación de madera}

Todas las ecuaciones desarrolladas establecen como criterio fundamental que la cubicación del fuste del árbol debe ser total, considerando su forma cilíndrica y sus variaciones diamétricas a lo largo del fuste. En realidad, la madera, cuando es aserrada, el primer proceso que sufre es que la troza es escuadrada; es decir, se le da una forma cuadrada, para luego procesarla según las dimensiones requeridas. Se sabe que estos bordes, llamados costillas, no son aprovechados como madera; incluye la corteza del árbol.

Es por lo que la diferencia entre el volumen estimado en pie y el volumen aserrado difiere significativamente, que puede llegar hasta el 40 \%. La ecuación propuesta parte del criterio que la cubicación de madera puede considerar la forma de bloque de madera, desde que se encuentra en rollo. El área se calcula simplemente con el diámetro al cuadrado, dividido entre dos. Esta ecuación proviene de aplicar el principio de Pitágoras, comparando el diámetro como la hipotenusa.

El desarrollo de la ecuación, según el principio de Pitágoras es la siguiente:

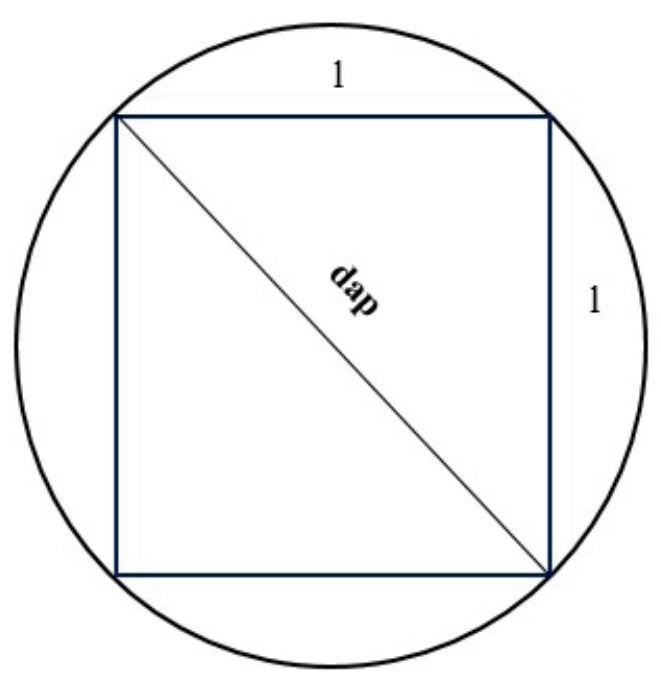

\begin{tabular}{|l|}
$\begin{array}{l}\text { Pitágoras } \\
(\text { dap })^{2}=(1)^{2}+(1)^{2} \\
(\text { dap })^{2}=2(1)^{2} \\
\frac{(\text { dap })^{2}}{2}=(1)^{2} \\
1=\frac{\text { dap }}{\sqrt{2}} \\
A=1 \times 1 \\
A=(\text { dap })^{2} \\
2 \\
A=\underline{D}^{2} \\
2\end{array}$ \\
\hline
\end{tabular}

Figura 1. Área aprovechable en una troza, simulando el diámetro como la hipotenusa de dos triángulos. Fuente: REDD/CCAD-GIZ, 2015.

Como resultado se tiene que la ecuación para calcular el volumen de madera de una troza es la siguiente:

\section{$V=(\underline{D / 100})^{2} \times L$}

\section{2}

\section{Donde:}

D es el diámetro de la troza en la cara menor, en centímetros.

L es la longitud estándar de una troza, en metros. 
Oriolus Revista Científica, ISSN: 2215-6100.1 (1). Julio, 2020, pp 27-38

Alpízar Vaglio

Si comparamos el volumen estimado a partir de la ecuación de área del círculo con la ecuación propuesta se obtendrá una importante diferencia, a continuación, un ejemplo:

$\mathrm{Dap}=40 \mathrm{~cm}$

Área del círculo $=(\underline{\text { dap }})^{2} \times \pi=(\underline{40})^{2} \times \pi=1256,6 \mathrm{~cm}^{2}$

$4 \quad 4$

Área con el método propuesto $=(\underline{40})^{2}=800 \mathrm{~cm}^{2}$

2

En este caso se obtiene una diferencia del $36 \%$.

En el caso del método al mecate se utiliza la circunferencia y la divide entre cuatro, lo que supone que la troza será escuadrada, pero hay una diferencia importante, considerado un error de estimación, cuando se compara con el método propuesto, que es cuando al final se escuadra la troza:

Área según PMT $=\left(\frac{C}{4}\right)^{2}$

Área según ecuación propuesta $=\underline{D}^{2}$

2

Prueba:

$\mathrm{Si} C=80 \mathrm{~cm}$, entonces $\mathrm{D}$ es igual a $25,5 \mathrm{~cm}$

El área de PMT sería $(80 / 4)^{2}=400 \mathrm{~cm}^{2}$

El área según la propuesta sería $\underline{(25,5)^{2}}=325,1 \mathrm{~cm}^{2}$

2

En este caso hay una diferencia del $19 \%$, en donde el volumen con PMT sobreestima el volumen por aprovechar.

\section{Variables por considerar en una ecuación de cubicación en pie}

Las ecuaciones usuales de cubicación de madera en árboles en pie parten del volumen del cilindro, ajustado por un factor de forma del fuste. Todas se desarrollan a partir de estudios en donde se cubica el volumen del fuste en secciones de un metro, cuando el árbol es cortado, de modo que se puede ajustar el volumen sumando cada segmento de fuste. Una variación del método, más ajustado, es correlacionar el volumen obtenido con una o dos variables de medición, la cual es ajustada por índices, llamados modelos alométricos.

La ecuación propuesta para la cubicación de madera en troza, también, podría aplicarse para cubicar madera en pie. Para ello es necesario considerar dos variables: la forma del fuste y la longitud.

\section{Forma de los fustes}

Los fustes de los árboles por lo general tienen una forma cilíndrica, cuyo diámetro disminuye o cambia según su altura. Esta forma, según Romahan y Ramírez (2012), varía con la especie y se llaman cuerpos dendrométricos; se clasifican en cuatro categorías: cilíndrico, cónico, paraboloide apolónico y neiloide, tal como lo muestra la figura 2.

Esta obra esta bajo una Licencia de Creative Commons Atribución NoComercial-SinDerivar Internacional 
Oriolus Revista Científica, ISSN: 2215-6100.1(1). Julio, 2020, pp 27-38

Alpízar Vaglio

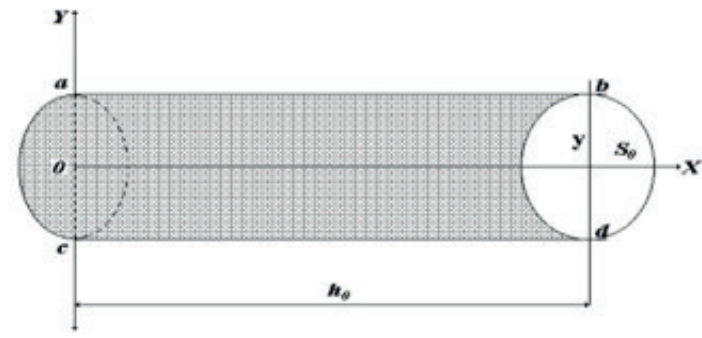

Cilindro

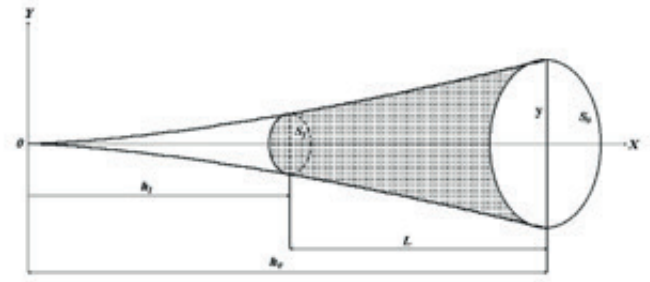

Neiloide

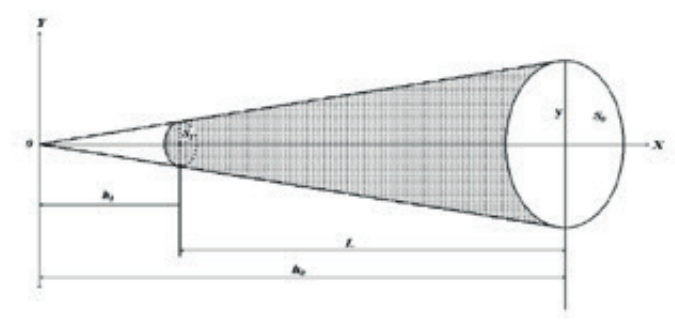

Cono

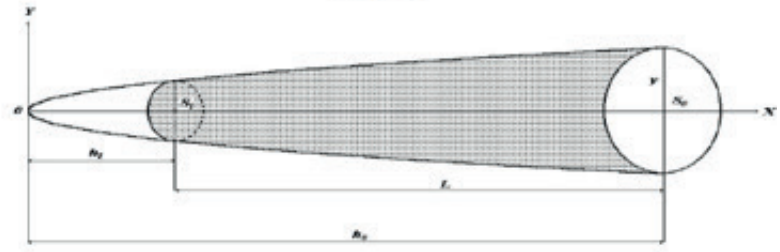

Paraboloide apolónico.

Figura 2. Tipos de formas de fustes. Fuente: Romahn y Ramírez, 2010.

CARDER (2013) clasifica algunas de las especies que se encuentran en el país, según la forma del fuste como lo muestra la tabla 1.

Cuadro 1. Forma de los fustes para algunas especies nativas de Costa Rica.

\begin{tabular}{ll}
\hline \multicolumn{1}{c}{ Especie } & \multicolumn{1}{c}{ Forma del fuste } \\
\hline Prioria copaifera Griseb. & Cilíndrico \\
Brosimum utile (H.B.K.) Pittier & Cilíndrico \\
Tabebuia rosea (Bertol.) A. DC. & Cónico \\
Anacardium excelsum (Kunth) Skeels & Cónico \\
Cedrela odorata L. & Paraboloide \\
Pterocarpus officinalis Jacq. & Neiloide \\
Campnosperma panamense Standl. & Paraboloide \\
Tapirira guianensis Aubl. & Paraboloide \\
Cordia alliodora (Ruiz \& Pav.) Oken & Neiloide \\
Jacaranda copaia (Aubl.) D. Don & Paraboloide \\
Carapa guianensis Aubl. & Neiloide \\
Guarea trichilioides L. & Paraboloide \\
Symphonia globulifera L. f. & Cónico \\
Otoba sp. & Cónico \\
\hline
\end{tabular}

Fuente: adaptado de CARDER, 2013.

Esta obra esta bajo una Licencia de Creative Commons Atribución NoComercial-SinDerivar Internacional 
Oriolus Revista Científica, ISSN: 2215-6100.1 (1). Julio, 2020, pp 27-38

Alpízar Vaglio

\section{Longitud}

La longitud está expresada en la altura del fuste. Sin embargo, una de las mayores complicaciones técnicas, en el caso de bosques, es calcular con certeza la altura de los árboles, puesto que la mayoría de los instrumentos de medición, llamados hipsómetros, funcionan con el principio de triángulos semejantes y es necesario separarse de los árboles a una distancia definida, lo cual no permite lograr una buena apreciación. Esta situación no es tan complicada en plantaciones forestales y en árboles aislados en potreros y sistemas agroforestales, pero en un bosque no es funcional.

La tecnología ha mejorado los instrumentos para estimar la altura de los árboles, existen los que utilizan un rayo láser y los que funcionan por radio onda, pero aún tienen limitaciones de uso. La ecuación propuesta propone desistir de estimar la altura de los árboles y en su lugar determinar el número de trozas que se obtienen de un fuste. Esto es más sencillo, porque la posibilidad de fallar en el número de trozas en menor, en comparación con utilizar una estimación en metros de altura.

Para ello es necesario definir un tamaño estándar de troza. La experiencia de los madereros es la troza de cuatro varas; aunque por efectos prácticos, el transporte de trozas puede ser de ocho y doce varas, lo común es que en el aserradero se procesen de cuatro varas; es decir, una longitud de 3,34 metros. También, la crisis de faltante de madera ha obligado que eventualmente se utilicen trozas menores a cuatro varas.

De acuerdo con Paniagua (s.f.) la longitud de las piezas de madera para construcción se consigue generalmente en múltiplos de $60 \mathrm{~cm}$ (dos pies), entre 1,80 y 7,20 metros. Sin embargo, dado que el mercado utiliza aún el sistema de la pulgada maderera tica, la cual tiene una extensión de cuatro varas, es usual que las trozas sean en dimensiones de cuatro varas y sus múltiplos.

Cuando la madera es aserrada, entre mayor es la longitud de la troza que se procesa mayor es la pérdida, por lo que una dimensión normal para el aserrío son las cuatro varas. En ocasiones, se pueden hacer cortes menores.

\section{Conclusiones y recomendaciones}

Es evidente que las ecuaciones de cubicación desarrolladas por las universidades no tienen aplicación práctica en el medio maderero nacional. Se continúa utilizando el método al mecate con unidades PMT, que utilizan varas y pulgadas, prohibidas por la Ley N. ${ }^{\circ} 5292$.

Los métodos de cubicación se basan en estimar el volumen total y parten de la ecuación del cilindro, cuando al final la madera es escuadrada en los aserraderos y se sobreestima la que se va a aprovechar realmente. La ecuación propuesta determina una diferencia importante con los métodos tradicionales y es de simple aplicación, puesto que parte del diámetro de la troza.

La ecuación propuesta no contradice las ecuaciones usuales del país, en el entendido que estas cubican la totalidad del volumen y pueden tener aplicaciones para estimar biomasa, pero no para estimar madera comercial.

La ecuación propuesta puede ser aplicada para cubicar árboles en pie. Para ello es necesario determinar un factor de reducción del diámetro, dado que la medición en campo se hace a partir del diámetro a la altura del pecho, que se encuentra en el extremo mayor. Es por lo que D debe

Esta obra esta bajo una Licencia de Creative Commons Atribución NoComercial-SinDerivar Internacional 
Oriolus Revista Científica, ISSN: 2215-6100.1 (1). Julio, 2020, pp 27-38

Alpízar Vaglio

ajustarse con un factor $f$, de modo que el diámetro por determinar es, precisamente, el que utilizará la troza una vez que es escuadrada.

El factor $\mathrm{f}$ debe determinarse a partir de una validación de campo, en donde se comparan los valores de volumen en pie y aserrado, utilizando diferentes factores f. Las formas de los fustes se clasifican en cuatro tipos, mencionados anteriormente. Es necesario hacer una validación en función de estos cuatro grupos, de modo que el factor f pueda aumentar o disminuir o mantenerse el diámetro medido a la altura del pecho. El factor $f$ es un ajuste en centímetros del D. De aquí se deduce que, para determinar el volumen de madera en pie con la ecuación propuesta, es necesario adicionarle el factor f, que es un ajuste al diámetro por concepto de variación, debido a la forma usual del fuste. En este caso la ecuación quedaría de la siguiente forma:

\section{$\mathrm{V}=\frac{\left(\frac{\mathrm{D}-f}{100}\right) 2}{2} \times \mathrm{L}$}

\section{Donde:}

D es el diámetro a la altura del pecho, en centímetros.

$f$ es el factor de ajuste por reducción del dap, conforme el fuste aumenta en altura.

L es la longitud estándar de una troza, en metros.

El factor $f$ se puede obtener realizando un estudio comparativo que estime el volumen en pie y se compare con el aserrado, aplicando la ecuación de cubicación de madera propuesta. Asimismo, es posible que este factor $f$ se pueda aplicar de forma estandarizada para grupos de especies, según la forma del fuste.

En cuanto a la longitud, se puede hacer un análisis de rendimiento, para determinar cuál debe ser el tamaño apropiado que provea el menor desperdicio o el mejor aprovechamiento de la troza. Es de esperar que entre más larga la troza, el desperdicio será mayor. En principio, se sugiere que dicha longitud sea de cuatro varas, convertido a metros (3,34 m), que es lo usual en el medio nacional.

Finalmente, para obtener el volumen de madera que se obtiene de un fuste, es importante sumar el volumen de cada troza estimada. Es necesario ajustar el factor $f$ para cada troza en el mismo fuste. Este factor, dependiendo de la forma del fuste, puede ser constante, de modo que se multiplica por dos para la segunda troza, por tres para la tercera y así sucesivamente; pero dependiendo del tipo de forma del fuste, puede aumentar y luego disminuir. Por lo tanto, es fundamental hacer una validación de campo de este factor, en función de las diferentes trozas que se obtienen del fuste.

\section{Bibliografía}

CARDER. Guía de cubicación de madera. Colombia: Unión Europea-CARDER (Corporación Autónoma Regional de Risaralda). Proyecto Posicionamiento de la Gobernanza Forestal de Colombia, (2013).

http://www.rivasdaniel.com/pdf/GUIA_DE_CUBICACION_MADERA.pdf

Esta obra esta bajo una Licencia de Creative Commons Atribución NoComercial-SinDerivar Internacional 
Oriolus Revista Científica, ISSN: 2215-6100.1(1). Julio, 2020, pp 27-38

Alpízar Vaglio

Chinchilla, O. y Gómez M. (2005). "Diferencias volumétricas en cuatro sistemas de medición de trozas de madera". Revista Ambientales, (2005): 76-82.

Costa Rica. Ley N. 5292, Sistema Internacional de Unidades. San José, CR: La Gaceta, 1973.

Costa Rica. Norma oficial para la comercialización de madera en troza y aserrada en condición verde. Decreto N¹2067-MEIC. Costa Rica: La Gaceta, 1980.

Dirección General Forestal. Guía para la elaboración de planes de manejo forestal. San José, C.R.: DGF, 1990.

Flores, J. Diagnóstico del sector industrial forestal. San José, CR: Editorial UNED, 1985.

Loján, L. "Una fórmula para estimar volúmenes en un bosque tropical húmedo". Revista Turrialba 16, N. ${ }^{\circ}$ 1. (1966): 67-72.

Muñoz, F. "Sistemas de medición utilizados en Costa Rica para calcular el volumen de madera en troza y aserrada". Kurú: Revista Forestal 3, N. 7 (2006).

Ortiz, E. (1989). Validación de ecuaciones de volumen para estimar volumen comercial para los árboles del bosque tropical. Nota Técnica 1, N. 3. Cartago, Costa Rica: ITCR, 2006.

Paniagua, V. Guía de uso y aplicaciones de la madera en la arquitectura en Costa Rica. San José, CR: Unidad de Investigación de la Escuela de Arquitectura, Universidad de Costa Rica. http:// www.arquis.ucr.ac.cr/assets/gu\%C3\%ADa-madera-de-usos-y-aplicaciones-de-la-madera-en-la-arquitectura-cr-encrypted.pdf

Prodán, M., Peters, R., Cox, F. y Real, P. Mensura forestal. Serie de Investigación y Educación en Desarrollo Sostenible. San José, CR: IICA-BMZ/GTZ (1997).

Programa REDD/CCAD-GIZ - SINAC. Inventario Nacional Forestal de Costa Rica 2014-2015. Resultados y Caracterización de los Recursos Forestales. San José, CR: Programa Reducción de Emisiones por Deforestación y Degradación Forestal en Centroamérica y la República Dominicana (REDD/CCAD/GIZ) y Sistema Nacional de Áreas de Conservación (SINAC) 2015.

Romahn, C.F. y Ramírez, H. Dendrometría. México: Universidad de Chapingo, 2010.

http://dicifo.chapingo.mx/licenciatura/publicaciones/dendrometria.pdf

Segura, M; Andrade, H. "¿Cómo construir modelos alométricos de volumen, biomasa o carbono de especies leñosas perennes? Turrialba, CR". Revista Agroforestería en las Américas N²6 (2008): 89-96.

SINAC. Guías de transporte de madera rolliza. 